\title{
P. Gascó
}

«El mundo es un lugar peligroso, no por las personas que son diabólicas sino por las personas que no hacen nada".

Albert Einstein

Éste fue el primer pensamiento que me vino a la cabeza al terminar de leer el magnífico artículo de I. Hernández-García, M.C. Sáenz-González y R. González-Celador sobre "Mortalidad atribuible al consumo de tabaco en España en el año 2006", que se publica en este número.

Efectivamente, Einstein con estas palabras mostraba su temor ante la pasividad y la escasa respuesta de un pueblo frente al problema que se avecinaba a la sociedad alemana previa a la Segunda Guerra Mundial.

Sociedades distintas pero alarmas encendidas hace años, quizás demasiados años, y éstas son cifras que dañan la vista y resquebrajan el corazón. Datos insoportables de asumir y es que la historia viene de largo.

Desde hace más de 60 años, los cigarrillos se identificaron con el cáncer de pulmón. Fueron dos importantes trabajos publicados de forma simultánea. El 27 de mayo de 1950, el Journal of the American Medical Association (JAMA) publicó el primer estudio que confirmaba la existencia de una posible asociación epidemiológica entre tabaco y cáncer de pulmón. El estudio se titulaba: «Fumar tabaco como posible factor etiológico en el carcinoma broncogénico: un estudio de 684 casos diagnosticados". Los autores, Wynder y Graham, constataron que el 96,5\% de los casos de cáncer de pulmón eran grandes fumadores. Cuatro meses después, dos ingleses, Richard Doll y Austin Bradford Hill, publicaron en el British Medical Journal (BMJ) un informe titulado

Comisión Asesora Técnica de Prevención y Control del Tabaquismo. Comunidad Foral de Navarra y Coordinador Nacional de Tabaquismo SemFYC.

\section{Correspondencia:}

Plácido Gascó

C.S. Iturrama

$\mathrm{C} /$ Serafin Olave, $\mathbf{s} / \mathbf{n}$

31080 Pamplona 
«El tabaco y el carcinoma de pulmón». En este trabajo pionero se estimaba que los riesgos relativos para los fumadores en comparación con los no fumadores eran de 6, 19, 26, 49 y 65 para exposiciones medias al tabaco de 3, 10, 20, 35 y 60 cigarrillos al día.

En esa época (1957) sucedieron dos hechos que llamaron poderosamente la atención de la sociedad de la epoca. La primera fue la muerte del fumador más importante de la historia del cine, Humphrey Bogart, y la segunda fue la muerte por cáncer del pulmón del Dr. Graham, fumador empedernido y curiosamente uno de los médicos que, siete años antes, había descubierto la relación entre el tabaco y su enfermedad.

La relación entre el consumo de tabaco y las principales enfermedades crónicas (enfermedades cardiovasculares, otros tipos de cáncer y enfermedades respiratorias crónicas, etc.) se fueron sucesivamente esclareciendo.

Los graves efectos nocivos del tabaco fueron oficialmente advertidos en el informe del Royal College inglés de 1962 y en el del Surgeon General de los EE.UU. en 1964.

En 1964 nuevos estudios pusieron de manifiesto la relación del tabaco con la enfermedad pulmonar obstructiva crónica. Tres años después se sugirió la implicación del hábito de fumar con el úlcus péptico y se comprobó también su relación con el cáncer de pulmón en la mujer.

En los años setenta la OMS (Organización Mundial de la Salud) advirtió a los países miembros que informaran a la población sobre los riesgos de consumo de tabaco.

En 1979 se documenta su relación con la enfermedad coronaria. A partir de 1980 se asocia con el ictus y con el cáncer de laringe, oral, de esófago y de vejiga.

Estudios recientes muestran un vínculo entre el tabaquismo y cataratas, artitris reumatoidea, impotencia, cáncer hepático, colorrectal, pancreático y renal en mujeres. Siguen aflorando abrumadoras evidencias científicas, de tal forma que las enfermedades relacionadas con el tabaco se han convertido en unos de los más importantes problemas de salud pública de nuestro tiempo.

El presente estudio que publica la revista Anales del Sistema Sanitario de Navarra tiene un gran interés y actualidad porque nos proporciona una estimación del número de muertes que causó el tabaquismo en España en 2006. Se trata de las muertes atribuibles (MA) al tabaco. También nos informa de cuáles son las causas por las que mueren nuestros fumadores y fumadoras (la causa específica de mortalidad) y de los años potenciales de vida perdidos atribuibles al consumo de tabaco (APVP).

El número total de fallecimientos atribuibles al tabaquismo en España se cifra según este estudio en 58.573 personas (2006). La estimaciones previas sobre mortalidad atribuidas al tabaco en España son del año 2004 y estimaban entonces 51.000 personas (Banegas, Díez-Gañán y col, 2006). Es decir, no sólo no se han conseguido reducir las muertes atribuibles al tabaquismo sino que han aumentado en 7.500 defunciones más. Este aumento de la mortalidad global se debe a la espectacular subida de los fallecimientos en mujeres, en las que se advertía sólo 
un ascenso suave pero continuado en los últimos años, en consonancia con la llegada a edades más avanzadas de las cohortes de mujeres con alta prevalencia de tabaquismo ya desde su vida adolescente y adulta.

Las estimaciones que aporta este estudio, a diferencia de estudios de mortalidad previamente publicados, pueden considerarse incluso conservadoras. Hasta la fecha, los estudios de mortalidad se han basado en el cálculo de riesgos relativos (RR) ajustados sólo por edad y sexo. Los autores de este estudio van más allá y utilizan RR ajustados además por otros factores de confusión, específicos para las diferentes patologías. Ésta es una de las fortalezas que aporta este estudio.

A estas cifras habría que añadir las muertes por tabaquismo pasivo, las muertes en edad infantil, otras neoplasias y muertes por incendios. Por tanto, si algo no se les podrá acusar a estos autores es de alarmismo. En todo caso, siempre debe tenerse en cuenta que el envejecimiento global de la población española requiere cierta cautela cuando se comparan los cambios absolutos en el número de defunciones con el tiempo.

Si estas cifras son correctas, y parece que lo son, estaríamos ante la mayor tasa de mortalidad atribuible al tabaco de la historia de España.

Si la monitorización de la mortalidad atribuible al tabaco nos ubica en qué fase de la epidemia tabáquica se encuentra España, está claro que estos datos rompen la tendencia decreciente de los últimos 10 años. Una forma de evidenciar el estancamiento entre las fases III y IV y reflejar los resultados de las políticas sembradas en los últimos años. El repunte en un $2 \%$ del número de fumadores, que comunica el avance de los datos de la Encuesta Nacional del Salud del 2008, parece reafirmar estos temores.

Casi todas las muertes relacionadas con el fumar (36\%) se originaron por neoplasias. El cáncer de pulmón sigue siendo en los varones la principal causa de muerte atribuible al tabaco y en las mujeres son las otras cardiopatías.

La salud de los ciudadanos es uno de los recursos más tangibles y productivos que un país puede tener. Este estudio demuestra que los años potenciales de vida perdidos por el tabaco en nuestros pacientes (APVP), sobre todo por muertes prematuras por cáncer de pulmón y por cardiopatía isquémica, siguen siendo muy altos.

Los costes económicos para los gobiernos, empleadores y el medioambiente incluyen gastos en seguridad social y salud, los costes por incendios y daños a edificios por la falta de cuidado al fumar; los costes al medioambiente; el absentismo laboral, la disminución de la productividad, el mayor número de accidentes y los mayores costes de las pólizas de seguro.

España tiene un sistema sanitario en el que predominan las políticas curativas frente a las preventivas. El no aprobar o demorar este marco legal verdaderamente adecuado y una política de precios -que imponga también barreras reales al consumo- desequilibra todavía más (si es posible) la balanza. También se echa en falta en España una iniciativa más decidida por parte de los sindicatos para defender a sus trabajadores del humo pasivo del tabaco en ámbitos como los bares, restaurantes y hostelería. ¿Dónde han estado los sindicatos en estos 
años? Si por algo han destacado ha sido precisamente por sus negligentes omisiones en la lucha contra el tabaquismo, que sin duda es el primer riesgo para la salud de sus trabajadores afiliados.

En pocos años habrá graves problemas para financiar los tratamientos oncológicos, porque superarán los 70.000 euros por paciente al año.

La OMS, el Banco Mundial y otros organismos internacionales advierten a sus miembros de la gravedad de la situación, indicando que la información no es suficiente para abordar el tema del tabaco, siendo necesarias medidas económicas y legislativas para enfrentarse a la epidemia.

Este estudio se publica en un momento en que se anuncia un nuevo aplazamiento para la aprobación de la ley de espacios sin humo.

Estos resultados deberían servir a los responsables sanitarios y políticos de revulsivo y atender a una sociedad cansada de esperar espacios libres de humo. El movimiento antitabaco y buena parte de la sociedad española, no cree que sea irrelevante el aplazar la aprobación de la ley aunque sean meses, teniendo en cuenta que cada día que pasa fallecen 161 personas por dicho hábito (124 hombres y 37 mujeres). Cuánta presión deben estar recibiendo los responsables de poner en marcha la ley que, ante hechos tan graves, deciden posponer su aprobación.

Si en España es apremiante la aprobación de una nueva ley, todavía más se necesita que se instrumentalicen todas las medidas necesarias para que una vez entre en vigor, su cumplimiento y seguimiento se cumplan sin negligencias ni ambigüedades.

Pero sólo con la ley de protección de espacios públicos libres de humo no es suficiente; la ley no puede entrar en los hogares privados donde sí puede colarse la concienciación y sensibilización. España posee el porcentaje más alto de fumadores que enciende sus cigarrillos en presencia de personas no fumadoras. También es de los países europeos que menos conciencia tiene de que respirar humo de tabaco sea perjudicial para su salud. Sólo Rumania, Bulgaria y Hungría nos superan (Eurobarómetro 2007).

A la vista de estos datos, está claro que queda mucho por hacer. No intervenir se podría entender como una mala praxis. Es imprescindible generar apoyo social a nuevas leyes y regulaciones y reiterar esfuerzos de sensibilización y educación del público de los efectos del tabaco, dejando claro que el fumador no es el problema a combatir.

Los partidos políticos deberían huir de la confrontación y de utilizar el tabaco como arma arrojadiza. La historia nos enseña que, cuando se da total prioridad a las decisiones ideológicas sobre las científicas, el resultado suele ser catastrófico.

España huele demasiado a humo ¿A qué esperan nuestros políticos para parar esa masacre? No busquen más excusas para aprobar este tipo de leyes. No debería temblarles el pulso. No hacerlo constituiría una ceguera e irresponsabilidad impropia de un país avanzado. 


\section{BIBLIOGRAFÍA}

1. Gately I. Tobacco: a cultural history of how an exotic plant seduced civilization. Grove Press, 2003. ISBN 0-8021-3960-4.

2. Banegas JR, Díez Gañán L, González Enríguez J, Villar Álvarez F, Rodríguez-Artalejo F. La mortalidad atribuible al tabaquismo comienza a descender en España. Med Clin (Barc) 2005; 124: 769-771.

3. Estapé J, Agustí A, Font A, Salvador T, Viñolas N. Tabaco y cáncer. Med Clin (Barc) 1987 (Supl. 1): 30-37.

4. Jiménez Ruiz C, González Vega JM, Escudero Bueno C, García Hidalgo A, Roca ToRRENT J, Solano Reina $S$ et al. Patología producida por el consumo de tabaco. En: Separ ed. Tabaquismo. Madrid 1995: 23-39

5. MarTínez-González MA, De Irala J. Medicina preventiva y fracaso clamoroso de la salud pública: llegamos mal porque llegamos tarde. Med Clin (Barc) 2005; 124: 656-660. 
